\title{
Negative emotions in the target speaker's voice enhance speech recognition under "cocktail-party" environments
}

\author{
Lingxi Lu ${ }^{1,2} \cdot$ Yu Ding ${ }^{1,2} \cdot$ Chuanwei Xue ${ }^{3}$ Liang $\mathrm{Li}^{1,2,3}$
}

Accepted: 9 September 2020 / Published online: 19 October 2020

(C) The Psychonomic Society, Inc. 2020

\begin{abstract}
Under a "cocktail-party" environment with simultaneous multiple talkers, recognition of target speech is effectively improved by a number of perceptually unmasking cues. It remains unclear whether emotions embedded in the target-speaker's voice can either improve speech perception alone or interact with other cues facilitating speech perception against a masker background. This study used two target-speaker voices with different emotional valences to examine whether recognition of target speech is modulated by the emotional valence when the target speech and the maskers were perceptually co-located or separated. The results showed that both the speech recognition against the masker background and the separation-induced unmasking effect were higher for the target speaker with a negatively emotional voice than for the target speaker with a positively emotional voice. Moreover, when the negative voice was fear conditioned, the target-speech recognition was further improved against speech informational masking. These results suggested that the emotionally vocal unmasking cue interacts significantly with the perceived spatial-separation unmasking cue, facilitating the unmasking effect against a masking background. Thus, emotional features embedded in the target-speaker's vocal timbre are also useful for unmasking the target speech in "cocktail-party" environments.
\end{abstract}

Keywords Cocktail-party problem $\cdot$ Speech unmasking $\cdot$ Vocal cue $\cdot$ Emotion $\cdot$ Perceived spatial separation

\section{Introduction}

In "cocktail-party" listening conditions (Cherry, 1953) when multiple talkers are speaking simultaneously, the targetspeech recognition can be improved by several perceptual unmasking cues (Arons, 1992; Schneider, Li \& Daneman, 2007). A speaker's vocal timbre conveys the "fingerprinting" characteristics with time-varying harmonic frequencies that help listeners instantly distinguish the target speaker even from a single spoken word (Pollack, Pickett \& Sumby, 1954). When listeners have prior knowledge of the target speaker's vocal timbre, selective attention to the target speaker

Liang Li

liangli@pku.edu.cn

1 School of Psychological and Cognitive Sciences, Peking University, Beijing 100080, China

2 Speech and Hearing Research Center, Key Laboratory on Machine Perception (Ministry of Education), Peking University, Beijing, China

3 Beijing Institute for Brain Disorders, Capital Medical University, Beijing, China is enhanced and recognition of the target speech is facilitated (Holmes, Domingo \& Johnsrude, 2018; Huang, Xu, Wu \& Li, 2010; Johnsrude et al., 2013; Yang et al., 2007).

In addition to the specific acoustic features of talkers' vocal timbres, emotional information is also embedded in the vocal "profiles" of the speaker. Previous studies (Dupuis \& PichoraFuller, 2010, 2014; Gordon \& Hibberts, 2011; Gordon \& Ancheta, 2017) have demonstrated that emotional information expressed by speech prosodies (how the speech is said) markedly modulates speech-word recognition even under noisy backgrounds. However, to our knowledge, little is known about the differences between the impact of emotional features that are embedded in a speaker's vocal timbre (who is saying) on the target-speech recognition under the noise energetic masking and that under the speech informational masking. Recently, Spreadborough and Anton-Mendez (2018) have evaluated the emotional valences of different singers' with different vocal timbres, and found that the performance for the emotional word judgment was impaired when the emotional valence of a song word was incongruent with the emotional valence of the singer's vocal timbre, indicating an interaction between the emotional processing of vocal timbre and the semantic processing of lyrics. Given that the vocal-timbre-based segregation is critical for 
releasing the attended speech from maskers (Bregman, 1994; Holmes et al., 2018; Huang et al., 2010; Johnsrude et al., 2013; Yang et al., 2007), an important question arises whether the emotional attributes of a target speaker's voice also influence target speech perception under adverse listening conditions. Filling this research gap is critical for enriching the understanding of how the emotions modulate the speech-in-masker perception, particularly under informational masking conditions.

Negative emotions with threaten-related signals play a crucial role in attracting attention under sensory competition conditions (Eastwood, Smilek \& Merikle, 2001; Fox, 2002; New \& German, 2015; Ohman, Flykt \& Esteves, 2001). Neuroimaging studies have also revealed the enhanced sensory processing for negative emotional prosodies compared to a neutral one under dichotic listening conditions (Grandjean et al., 2005; Sander et al., 2005). Based on the view of prioritized processing for threaten-related negative emotions (Vuilleumier, 2005), our additional assumption is that a target speech stream spoken with a negative vocal timbre can be better perceptually tracked and recognized against interfering backgrounds than the speech spoken by voices in other categories of emotional valences.

Previous studies have shown that learned knowledge about a target speaker's vocal timbre influences speech perception. For example, perceptual learning of a talker's voice can promote perception of the speech read by this voice (Case, Seyfarth \& Levi, 2018; Levi, Winters \& Pisoni, 2011; Nygaard \& Pisoni, 1998). Moreover, in our previous studies, we found that emotional conditioning of a target voice improves speech intelligibility associated with that voice under noisy masking conditions ( $\mathrm{Lu}$ et al., 2018). However, the Lu et al. study (2018) has not addressed the question about whether and how emotional features embedded in speakers' vocal timbres affect the speech-in-noise recognition. Specifically, in the Lu et al. study (2018), one target speaker's voice was aversively conditioned in the experimental group, and the speech-recognition performance with that voice was better than that in the control group who received neutral learning of the same target voice. To further this line of studies, the present study applied the emotional conditioning paradigm to intensify the emotional characteristics attached to different speakers' vocal timbres, and investigated how the subjective emotional feelings induced by different speaker's vocal timbres after conditioning modulate the speech-unmasking process.

To perceptually segregate target speech from maskers in a complex auditory background, listeners take advantage of various unmasking cues. One of the most important unmasking cues is the perceived spatial separation between the target speech and maskers, which largely facilitates the perceptual segregation of the target voice stream from the competing background streams, and enhances the target-speech recognition especially in a speech (informational) masking condition compared to a noise (energetic) masking condition (Arbogast, Mason \& Kidd, 2002; Freyman, Helfer, McCall \& Clifton,
1999; Li, Daneman, Qi \& Schneider, 2004; Wu, Wang, Chen, Qu \& Li, 2005). Under the noise-masking condition, the energetic masking effect occurs at the peripheral level and the target auditory signal is physically degraded by the noise. For speech informational maskers, the competing auditory streams bring in additional non-energetic masking (i.e., informational masking), which occurs at the perceptual and cognitive levels (Brungart, 2001; Freyman et al., 1999; Freyman, Balakrishnan \& Helfer, 2001, 2004; Schneider et al., 2007). Perceived spatial separation between the target and the maskers helps listeners allocate their spatial attention to the target voice stream, leading to a significant release of target speech from informational masking. In the Lu et al. study (2018), an additive unmasking effect of learned emotion was identified in addition to the effect of the perceived spatial separation on speech perception under two-talker speech masking. Given that the target speaker's vocal timbre contributes to the perceptual segregation between the target speech and the competing masker (Bregman, 1994), and that the perceived spatial-separation-induced unmasking is largely dependent on the masking type (informational versus energetic masking) (Arbogast et al., 2002; Freyman et al., 1999; Huang, Huang, Chen, Wu \& Li, 2009; Li, Kong, Wu \& Li, 2013; Li et al., 2004; Wu et al., 2005), it is of importance to investigate the interactions between the vocal emotion and perceived spatial separation under different types of masking stimuli.

In summary, to advance the understanding of the psychological mechanisms involving emotional processes in solving the cocktail-party problem, the present study applied an emotional conditioning paradigm and examined the unmasking effect of emotional vocal timbres and its relationship with spatiallyinduced unmasking on speech perception under either the twotalker speech-masking condition or the steady-state speechspectrum-noise-masking condition. When a negative emotion becomes one of the salient features of the target speech (especially through fearing conditioning), it becomes an unmasking cue, attracting more selective attention to the target-speech stream and facilitating recognition of the target speech against a masker, particularly an informational masker. Our hypothesis is that the negative emotion induced by the speaker's vocal timbre and intensified by aversive conditioning can enhance the targetspeech recognition in the cocktail-party environment, and the emotional and spatial unmasking cues interact to each other in facilitating the speech-in-noise perception.

\section{Methods}

\section{Participants}

Twenty-four Mandarin-speaking university students (16 females, eight males) between 18 and 26 years old (mean \pm $\mathrm{SD}, 21.4 \pm 2.2$ years) participated in this study. They were 
right-handed and had normal pure-tone hearing thresholds (no more than $25 \mathrm{~dB}$ HL for each ear) and bilaterally symmetric hearing (difference no more than $15 \mathrm{~dB}$ between the two ears) between 0.125 and $8 \mathrm{kHz}$. All the participants gave their informed consent before the experiment. They were paid a modest stipend for their participation. The procedures of this study were approved by the Committee for Protecting Human and Animal Participants of the School of Psychological and Cognitive Sciences at Peking University.

\section{Apparatus and materials}

The participant was seated in a sound-attenuated room. The acoustical signals were digitized at the sampling rate of 22.05 $\mathrm{kHz}$, transferred from the PCI Express Sound Blaster X-Fi Xtreme Audio (Creative Technology Ltd, Singapore) and binaurally presented to participants using Sennheiser (Hanover, Germany) HD 265 Linear headphones. The presentation of acoustic stimuli was controlled by a Psychtoolbox interface (Brainard, 1997 based on Matlab software (MathWorks Inc. Natick, MA, USA). The sound-pressure level (SPL) of the target speech was $56 \mathrm{~dB}$ SPL at each ear. Calibration of the sound level was conducted using the Audiometer Calibration and Electroacoustic Testing System (AUDit and System 824, Larson Davis, USA). The SPL of the masker was adjusted to generate four signal-to-noise (masker) ratios (SNRs) of $0 \mathrm{~dB}$, $4 \mathrm{~dB},-8 \mathrm{~dB}$, and $-12 \mathrm{~dB}$.

The speech stimuli were Chinese nonsense sentences, which were syntactically correct but not semantically meaningful (Huang et al., 2009; Li et al., 2013; Wu et al., 2005; Yang et al., 2007). The English translations of the sentences were similar but not identical to the English nonsense sentences that were developed by Helfer (1997) and used in previous studies (e.g., Freyman et al., 1999; Li et al., 2004). Each sentence has 12 syllables (also 12 characters) including three keywords (the subject, predicate, and object) with two syllables for each. For instance, the English translation of a Chinese nonsense sentence was "This polyester will expel that stomach" (keywords underlined). In nonsense sentences, there is no contextual support for recognizing each of the keywords. The development of the Chinese nonsense sentences was described elsewhere (Yang et al., 2007).

The target speech sentences were spoken by each of the two younger-adult female talkers (Voice 1 and Voice 2) who were instructed to read with a neutral prosody. The mean fundamental frequency of the two voices was $236 \mathrm{~Hz}$ and $244 \mathrm{~Hz}$, respectively, retrieved using the customized script of Praat software version 6.0.36 (www.praat.org). Fifty young students ( 25 females, 25 males) between 19 to 28 years old (mean $\pm \mathrm{SD}, 23.0 \pm 2.5$ years) were invited to rate the scores of emotional feelings induced by the vocal timbre of the two voices using the 9-point SAM scales for the emotional valence (1, most unpleasant; 5 , neutral; 9 , most pleasant) and emotional arousal (1, lowest; 5, moderate; 9, highest) (Bradley \& Lang, 1994). Each of the two voices was presented five times (five different sentences) and the order of the presentation of the ten sentences was arranged randomly for each of the raters. The average scores of emotional valence and emotional arousal were obtained for each individual rater. These raters did not participate in the formal experiment. The emotional feelings induced by the vocal timbre of the two target voices were (mean $\pm \mathrm{SD}$ ): Voice 1, emotional valence $=4.34 \pm 1.40$, emotional arousal $=4.88 \pm$ 1.50; Voice 2, emotional valence $=6.09 \pm 1.62$, emotional arousal $=4.88 \pm 1.59$. A one-sample $t$-test showed that the emotional valence of Voice 1 was significantly lower than the middle (neutral) value of $5(t(49)=-3.33, p<.01$, Cohen's $d=$ $.47)$, and that of Voice 2 was significantly higher than the value of $5(t(49)=4.75, p<.001$, Cohen's $d=.67)$. Also, the paired-sample $t$-test showed that the emotional valence of Voice 1 was significantly more negative than that of Voice 2 $(t(49)=-5.18, p<.001$, Cohen's $d=1.16)$. No significant difference was found between the emotional arousal of Voice 1 and that of Voice $2(t(49)=.05, p=.96$, Cohen's $d=.01)$. Therefore, we labeled Voice 1 as the "unpleasant voice" and Voice 2 as the "pleasant voice" according to the emotional valence of their vocal timbres obtained from the 50 raters.

The speech masker was a 60-s loop of digitally combined continuous recordings of Chinese nonsense sentences spoken by two other female talkers with the mean fundamental frequency of $239 \mathrm{~Hz}$ and $269 \mathrm{~Hz}$, respectively. The sound pressure levels were the same across the two masking talkers' speech sounds. All of the masking sentences were also spoken in a neutral prosody. Each of the two target talkers and the two masking talkers spoke different sentences. The keywords of masking sentences did not appear in any of the target sentences. The noise masker was 10 -s steady-state speechspectrum noise, containing a mixture of 113 sentences voiced by 50 female younger-adult speakers at a medium rate (including 57 sentences spoken by 25 speakers and 56 sentences spoken by other 25 speakers) (Yang et al., 2007).

In the experiment, the emotional conditioning paradigm was applied to intensify the emotional characteristics that were attached to individual speakers' vocal timbres. The unconditioned aversive sound and neutral sound used for the conditioning in this study were adopted from the $\mathrm{Lu}$ et al. study (2018). The intensely aversive sound (unconditioned stimulus, US) was a loud female screaming from the database of the International Affective Digitized Sounds (IADS) (Bradley \& Lang, 2007) with a peak SPL of $80 \mathrm{~dB}$ and a duration of 4.09 s. This screaming sound induced aversive feelings according to self-reports of the 24 participants. After listening to the aversive stimulus, the 9-point SAM ratings for all the participants confirmed the effectiveness of the aversive stimulus in inducing negative feelings (emotional valence $=1.89 \pm 1.11$; emotional arousal $=7.29 \pm 1.31$ ). In addition, a 6 -s sound of 
train whistling obtained from the IADS database with a peak sound-level pressure of $60 \mathrm{~dB}$ SPL was chosen as the emotionally neutral (control) sound, which had neutral emotional valence $(5.37 \pm 1.49)$ and moderate emotional arousal (4.42 \pm 1.90 ) across to 24 participants' SAM ratings.

The perceived spatial laterality of the speech stimuli was manipulated by setting different values of the interaural time differences (ITDs). The target sentence was presented always with the right ear leading the left ear by $2 \mathrm{~ms}$. Thus, due to the auditory precedence effect (Wallach, Newman \& Rosenzweig, 1949), listeners perceived the target speech as coming from the right ear. Under the condition of perceived co-location, the masker was also presented with the rightleading ITD of $2 \mathrm{~ms}$ and perceived as coming from the same ear (right ear) as the target. Under the condition of perceived separation, the masker was presented with the left-leading ITD of $2 \mathrm{~ms}$ and perceived as coming from the left ear. Note that shifts between the two (perceived co-location, perceived separation) conditions did not change either the SNR or the diffuseness/compactness of the sound images.

\section{Procedure}

There were four within-participant variables: (1) vocal timbre (pleasant, unpleasant), (2) perceived laterality relationship (separation, co-location), (3) SNR (0 dB, $-4 \mathrm{~dB},-8 \mathrm{~dB},-12$ $\mathrm{dB}$ ) and (4) masker type (speech, noise). For each participant, there were $32(2 \times 2 \times 4 \times 2)$ conditions, and 15 trials $(15$ sentences) for each condition. Here, the factor of SNR was introduced for calculating the speech-recognition thresholds (see the Results section). Considering the time duration of the entire experiment (about $180 \mathrm{~min}$ ), the 32 conditions were divided into two sessions based on the two masker types, each session containing 16 conditions. Participants participated in the two sessions in 2 days, and the order of the two sessions was counterbalanced across participants. For each session, four blocks of tests were used for the $2 \times 2$ conditions of perceived spatial laterality and vocal timbre. The presentation order of four blocks was Latin-square balanced across participants, and the order of 60 trials of four SNRs in each block was arranged randomly. Figure 1 displays the experimental procedure for each session.

There was one between-participant variable: conditioning type (CS+, CS-). The 24 participants were randomly allocated into two groups. For half of the participants (group 1), the unpleasant voice was conditioned with the aversive sound (unpleasant $\mathrm{CS}+$ ), while the pleasant voice was paired with the neutral (control) sound (pleasant CS-). For the other half of the participants (group 2), the pleasant voice was aversively conditioned (pleasant $\mathrm{CS}+$ ) and the unpleasant voice was neutrally learned (unpleasant CS-).

In each trial, the participant pressed a response button to start the masking sound, and $1 \mathrm{~s}$ later a target sentence spoken by one of the two target voices was presented along with the masker. The masker stopped at the same time as the target sentence. The participant's task was to repeat the target sentence as best as he/she could. Two experimenters scored whether each of the two syllables for each of the three keywords (in a target sentence) had been identified correctly. At the beginning of each block, two additional trials with 0 SNR were presented to help the participant identify which of the two target speakers will be presented in that block. These two trials were excluded from data analyses.

Before the formal testing, all the participants received a practice session to ensure they fully understood the procedures and performed the task correctly. The target sentences used in the training did not appear in the formal testing. After the practice, two sessions of emotional learning were carried out for each participant. The first session with 42 trials (21 times repeated for each CS+ and CS- condition) was carried out before the initial speech-recognition testing, and the second session of 14 trials (seven times repeated for each CS+ and CS- condition) was carried out after two blocks of speechrecognition testing to strengthen the conditioning effect. For each conditioning trial, the voice was first presented, followed by the US or the neutral sound immediately. The inter-trial interval was between $2 \mathrm{~s}$ and $3 \mathrm{~s}$ with a random value. Participants were instructed to listen to the sounds without any tasks during the sessions of emotional learning.

At the end of the experiment, participants were instructed to rate their subjective emotional feelings induced by the vocal timbre of the two target voices and that induced by the aversive/neutral sounds using the 9-point SAM scales (Bradley \& Lang, 1994). Each of them was repeated for five times, and the averaged emotional valence and emotional arousal were calculated for each individual participant. The ten randomly ordered trials of the two voices were first presented, followed by ten randomly ordered trials of aversive/ neutral sounds.

\section{Results}

\section{Recognition accuracy}

To calculate the speech-recognition threshold, a logistic psychometric function

$y=\frac{1}{1+\mathrm{e}^{-\sigma(x-\mu)}}$

was fitted to each participant's recognition accuracy, using the Levenberg-Marquardt method (Wolfram, 1991), in which $x$ represents the SNR, $y$ represents the recognition accuracy of keywords under the corresponding SNR, $\sigma$ is the slope of the psychometric function, and $\mu$ is the SNR corresponding to 


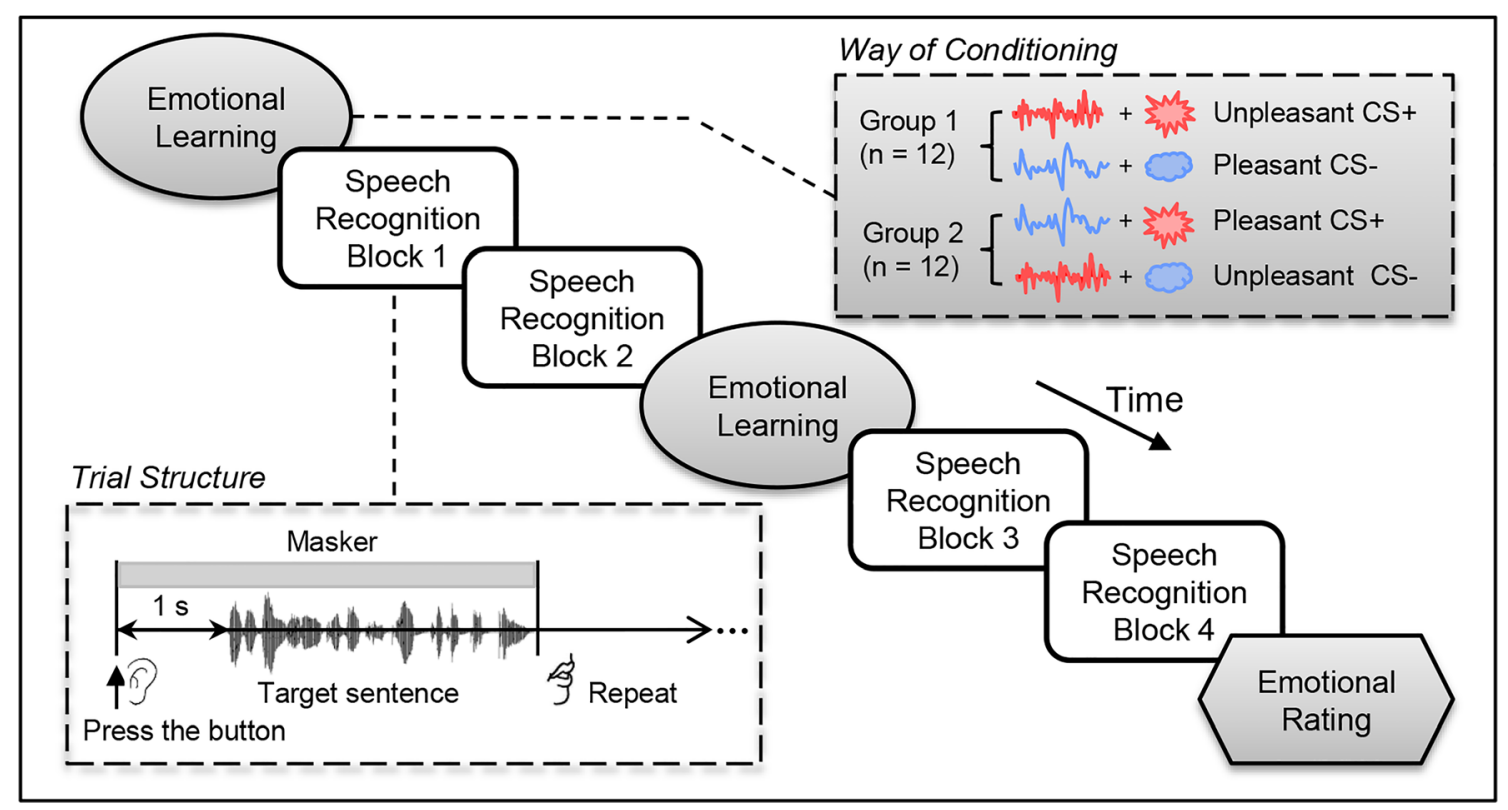

Fig. 1 Experiment procedures. Participants received two sessions of emotional learning and four blocks of speech recognition tests, followed by the final session of emotional rating. During emotional learning, the pleasant and unpleasant voices were paired with both aversive (CS+) and neutral (CS-) sounds. In speech recognition task, the participants listened to a target sentence under masking and repeated the speech he or she had listened to
$50 \%$ correct on the psychometric function.

Figure 2 displays the group-mean correct identification of the keywords in the target sentence under all the conditions, along with the group-mean best-fitting psychometric function. Each participant's speech-recognition threshold $(\mu)$ was obtained for further analyses.

\section{The speech-recognition threshold under the speech- masking condition}

A 2 (vocal timbre: unpleasant, pleasant) $\times 2$ (perceived laterality relationship: co-location, separation) $\times 2$ (way of conditioning: unpleasant CS+ and pleasant CS-, unpleasant CS- and pleasant CS+) three-way ANOVA for the speechrecognition threshold $(\mu)$ under the two-talker speechmasking condition showed that the main effect of vocal timbre was significant $\left(F(1,22)=32.79, p<.001, \eta_{p}{ }^{2}=.60\right)$, the main effect of perceived laterality relationship was significant $\left(F(1,22)=380.94, p<.001, \eta_{p}{ }^{2}=.95\right)$, and the main effect of way of conditioning was significant $(F(1,22)=8.75, p=$ $\left..007, \eta_{p}{ }^{2}=.29\right)$. In addition, there was a significant interaction between vocal timbre and way of conditioning $(F(1,22)=$ $14.00, p=.001, \eta_{p}{ }^{2}=.39$ ), and a significant interaction between vocal timbre and perceived laterality relationship $\left(F(1,22)=17.61, p<.001, \eta_{p}{ }^{2}=.45\right)$.

Improved speech recognition for the unpleasant voice Figure 3 shows simple effects of the two-way interactions. For each of the target voices, the factor of conditioning was the between-participant variable. That is, for half of the participants the target voice was aversively conditioned (CS+), while for the other half of the participants it was neutrally learned (CS-). Therefore, separate two-way ANOVAs (perceived laterality relationship $\times$ conditioning type) were conducted for the two target voices. As is shown in the upper panels of Figure 3, for the unpleasant voice, the main effects of perceived laterality relationship $(F(1,22)=209.53, p<$ $\left..001, \eta_{p}{ }^{2}=.91\right)$ and conditioning $(F(1,22)=13.25, p=.001$, $\left.\eta_{p}{ }^{2}=.38\right)$ were significant. No interaction was found between these two variables $(p=.145)$. Post hoc contrasts showed that speech recognition of the unpleasant voice was significantly better when the target and the maskers were perceptually separated compared to when they were perceptually co-located $(t(23)=14.09, p<.001$, Cohen's $d=2.88$, paired-sample $t$-test). Also, by pairing the unpleasant voice with the intensively negative emotion (CS+), the recognition threshold of the target speech articulated by unpleasant voice was lower than its CS- controls $(t(22)=-3.64, p=.001$, Cohen's $d=$ 1.49 , independent-sample $t$-test). For the pleasant voice, the ANOVA analysis showed a significant main effect of perceived laterality relationship $\left(F(1,22)=186.57, p<.001, \eta_{p}{ }^{2}\right.$ $=.90$ ). The main effect of conditioning type reached only marginal significance $(p=.088)$, and the interaction between the two variables was not significant $(p=.159)$.

In conclusion, these results showed that negatively conditioning an unpleasant voice, but not a pleasant voice, improved the recognition of the target speech spoken by that voice. 

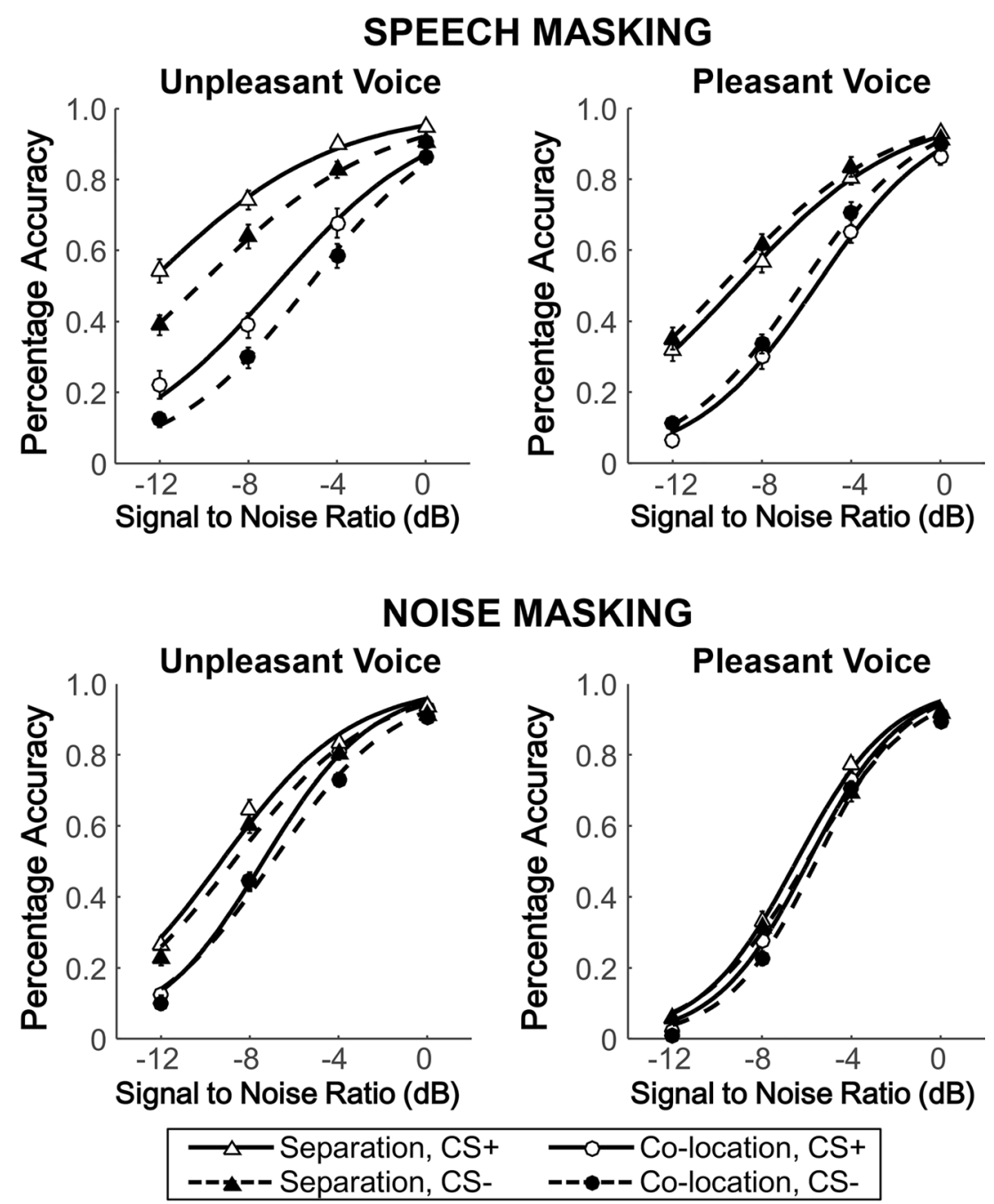

Fig. 2 Group-averaged recognition accuracy and the best-fitting psychometric function under all the conditions under speech and noise masking. Solid lines represent the CS+ conditions and dashed lines represent the CS- conditions. Triangles represent the separation conditions and circles

Larger spatial release from masking for the unpleasant voice As is shown in the lower panels of Figure 3, for the emotional learning (CS+) condition, a two-way ANOVA analysis (vocal timbre $\times$ perceived laterality relationship) revealed a significant main effect of vocal timbre $\left(F(1,22)=20.06, p<.001, \eta_{p}{ }^{2}\right.$ $=.48)$, a significant main effect of perceived laterality relationship $\left(F(1,22)=191.96, p<.001, \eta_{p}{ }^{2}=.90\right)$, and a significant two-way interaction $\left(F(1,22)=18.44, p=.002, \eta_{p}{ }^{2}=\right.$ .36). Separate independent $t$-tests showed that the speech recognition for the unpleasant voice was better than that for the pleasant voice either when the target and maskers were colocated $(t(22)=2.28, p=.033$, Cohen's $d=.93)$ or when they were separated $(t(22)=5.08, p<.001$, Cohen's $d=2.07)$. The simple effect of the interaction revealed a larger spatial release from masking (the amount of decreased speech-recognition threshold from co-location to separation) for the unpleasant voice compared to the pleasant voice $(t(22)=3.53, p=.002$, represent the co-location conditions. The recognition accuracy was increased from the SNR of $-12 \mathrm{~dB}$ to $0 \mathrm{~dB}$ for both the pleasant and the unpleasant vocal timbre. Speech-recognition threshold $(\mu)$ at the 0.5 correct level was obtained for each participant

Cohen's $d=1.44)$. For the control learning (CS-) condition, the same ANOVA analysis revealed a significant main effect of perceived laterality relationship $(F(1,22)=199.13, p<$ $\left..001, \eta_{p}{ }^{2}=.90\right)$ and a significant interaction between vocal timbre and perceived laterality relationship $(F(1,22)=5.15, p$ $\left.=.033, \eta_{p}{ }^{2}=.19\right)$, but not a significant main effect of vocal timbre $(p=.670)$. The simple effect of the interaction showed a larger spatial release from masking for the unpleasant voice compared to the pleasant voice $(t(22)$ $=2.27, p=.033$, Cohen's $d=.93$ ), which was similar to the result of the $\mathrm{CS}+$ condition. However, in contrast to that under the CS+ condition, under the CS- control condition when the unpleasant voice and the pleasant voice were neutrally learned, there was no significant differences between the two voices when the target and maskers were either perceptually co-located ( $p=$ $.052)$ or separated $(p=.433)$. 

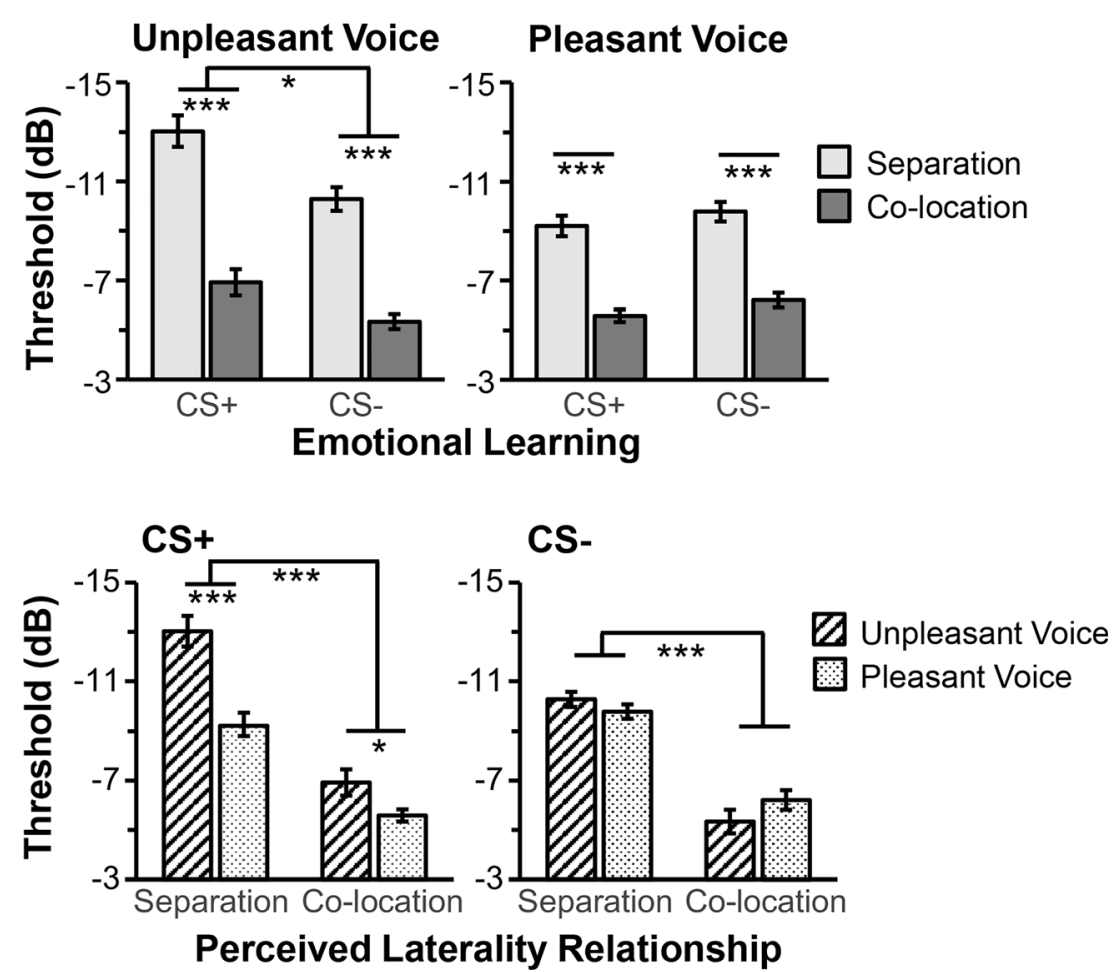

Fig. 3 Comparison of the speech-recognition thresholds between the pleasant and unpleasant voices under speech masking. Perceived spatial separation increased the speech recognition for both voices and the aversive conditioning enhanced the speech recognition for the unpleasant

In conclusion, these results suggested that under the speech-masking condition, negatively conditioning the target voice improved the speech recognition more largely for an unpleasant target voice compared to a pleasant one. Moreover, the spatial release of target speech from masking was larger for an unpleasant voice than a pleasant one regardless of the conditioning manipulation.

\section{The speech-recognition threshold under the noise- masking condition}

A three-way (vocal timbre by perceived laterality relationship by conditioning type) ANOVA for the speech-recognition thresholds $(\mu)$ under the noise-asking condition showed a significant main effect of vocal timbre $(F(1,22)=282.48, p<$ $\left..001, \eta_{p}{ }^{2}=0.93\right)$, a significant main effect of perceived laterality relationship $\left(F(1,22)=139.01, p<.001, \eta_{p}{ }^{2}=\right.$ $0.86)$, but no main effect of conditioning type $(p=0.96)$. In addition, there was a significant interaction between vocal timbre and way of conditioning $(F(1,22)=13.62, p=.001$, $\left.\eta_{p}{ }^{2}=.38\right)$, and a significant interaction between vocal timbre and perceived laterality relationship $(F(1,22)=57.65, p$ $<.001, \eta_{p}{ }^{2}=.72$ ).

Disappearance of unmasking effect of aversive conditioning for the unpleasant voice To untangle the interactions, separate voice (upper panel). The speech recognition for the unpleasant voice was better than the pleasant voice under CS+ condition but not CScondition (lower panel). Error bars represent standard errors (SEs). ${ }^{*} p<.05,{ }^{* * * *} p<.001$

two-way ANOVAs (perceived laterality relationship $\times$ conditioning type) were conducted for each target voice. As is shown in the upper panels of Figure 4, the main effect of perceived laterality relationship was significant (for the unpleasant voice, $F(1,22)=222.24, p<.001, \eta_{p}{ }^{2}=.91$; for the pleasant voice, $\left.F(1,22)=9.88, p=.005, \eta_{p}{ }^{2}=.31\right)$. The speech recognition of the unpleasant voice was better when the target and the maskers were perceptually separated compared to when they were perceptually co-located (for the unpleasant voice, $t(23)=15.15, p<.001$, Cohen's $d=3.09$; for the pleasant voice, $t(23)=3.20, p=.004$, Cohen's $d=.65)$. The main effect of conditioning type was not significant for the unpleasant voice $(p=.139)$, and reached the marginal significance for the pleasant voice $(p=.050)$. The interaction between the two variables was not significant for both the unpleasant and the pleasant voices ( $p=.605$ and .655 , respectively).

In summary, compared to the results of speech-recognition threshold under the speech-masking condition, the unmasking effect of the aversive conditioning of the unpleasant voice disappeared under the noise-masking condition.

Larger spatial release from masking for the unpleasant voice As is shown in the lower panels of Figure 4, for the emotional learning (CS+) condition, a two-way ANOVA analysis (vocal timbre $\times$ perceived laterality relationship) revealed a significant main effect of vocal timbre $\left(F(1,22)=56.40, p<.001, \eta_{p}{ }^{2}\right.$ 

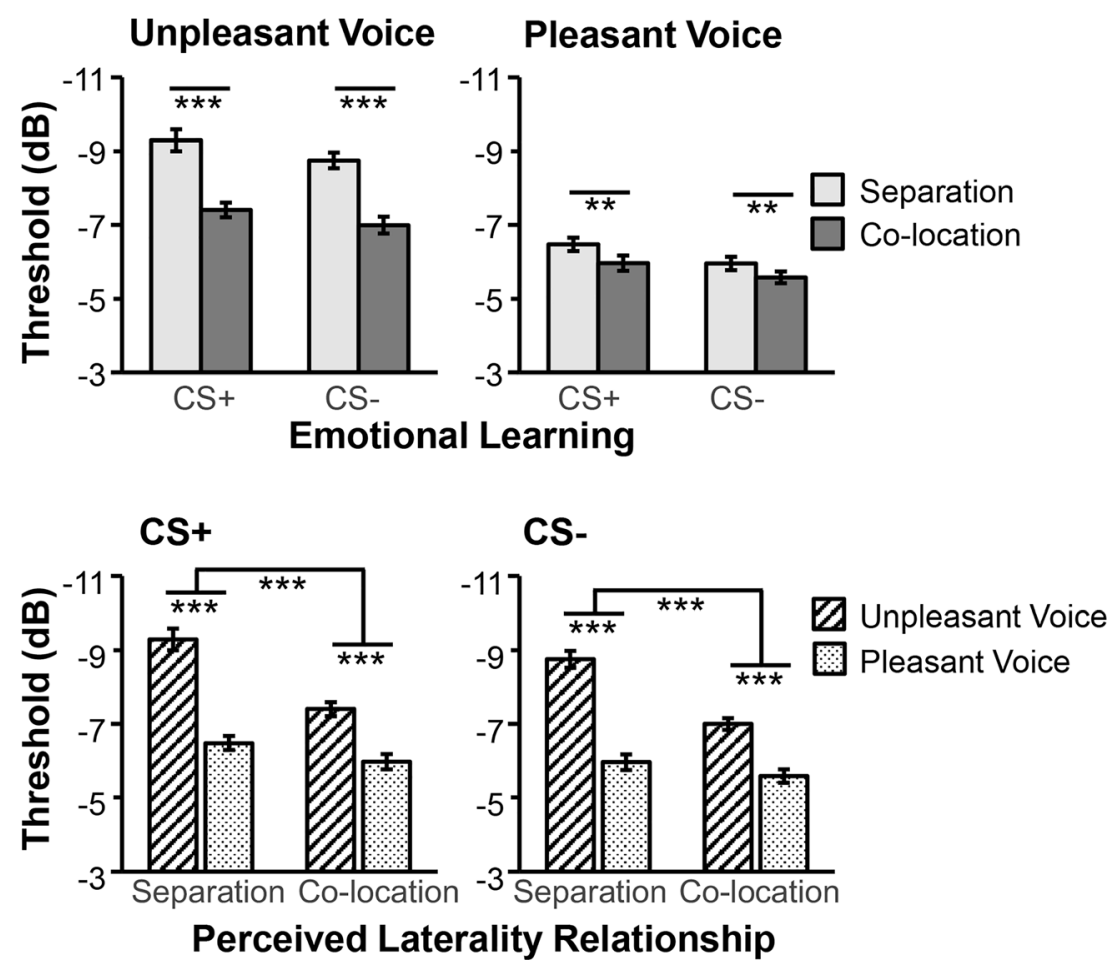

Fig. 4 Comparison of the speech-recognition thresholds between the pleasant and unpleasant voices under noise masking. Perceived spatial separation increased the speech recognition for both voices (upper

$=.72)$, a significant main effect of perceived laterality relationship $\left(F(1,22)=68.92, p<.001, \eta_{p}{ }^{2}=.76\right)$, and a significant two-way interaction $\left(F(1,22)=22.81, p<.001, \eta_{p}{ }^{2}=\right.$ $.51)$. Separate independent $t$-tests showed that the speechrecognition performance of the unpleasant voice was better than that of the pleasant voice either when the target and maskers were co-located $(t(22)=5.01, p<.001$, Cohen's $d$ $=2.05)$ or when they were perceptually separated $(t(22)=$ $8.15, p<.001$, Cohen's $d=3.33$ ). The simple effect of the interaction showed a larger spatial release of target speech from masking for the unpleasant voice compared to the pleasant voice $(t(22)=4.78, p<.001$, Cohen's $d=1.95)$. For the control learning (CS-) condition, the same ANOVA analysis also showed a significant main effect of vocal timbre $(F(1,22)$ $\left.=69.52, p<.001, \eta_{p}{ }^{2}=.76\right)$, a significant main effect of perceived laterality relationship $(F(1,22)=80.94, p<.001$, $\left.\eta_{p}{ }^{2}=.79\right)$, and a significant interaction between the two variables $\left(F(1,22)=33.59, p<.001, \eta_{p}{ }^{2}=.60\right)$. Increased speech recognition of the speech articulated by the unpleasant voice than by the pleasant voice was observed under both the colocation condition $(t(22)=5.05, p<.001$, Cohen's $d=2.06)$ and the separation condition $(t(22)=10.07, p<.001$, Cohen's $d=4.11)$. Similar to the results of CS+ condition, unpleasant voice also had a larger spatial release from masking than the pleasant voice $(t(22)=5.80, p<.001$, Cohen's $d=2.37)$.

Taken together, these results suggested that, under the noise masking condition, both the speech-recognition panel). The speech recognition for the unpleasant voice was better than the pleasant voice under both the CS+ condition and the CS- condition (lower panel). Error bars represent SEs. ${ }^{* *} p<.01,{ }^{* * * *} p<.001$

performance was better and the spatial-induced unmasking effect was larger for the unpleasant voice than the pleasant voice, regardless of the emotional learning type.

\section{Spatial release of target speech from masking}

In the analysis mentioned above, the speech-recognition performance was investigated under either the speech-masking or the noise-masking condition. In the next step, the results of target speech-recognition performance under the speechmasking condition and that under the noise-masking condition were combined. To simplify the analysis, the amount of spatial release from masking was first calculated, which reflected the benefits of perceived spatial separation between target and maskers on the target-speech recognition. Next, a three-way ANOVA analysis (vocal timbre $x$ way of conditioning $x$ masking type) was conducted for the spatial release amount.

As is shown in Figure 5, the main effect of vocal timbre was significant $\left(F(1,22)=36.06, p<.001, \eta_{p}{ }^{2}=.62\right)$, and the main effect of masking type was significant $(F(1,22)=$ $\left.185.96, p<.001, \eta_{p}{ }^{2}=.89\right)$. The main effect of conditioning was not significant $(p=.288$ ). A larger spatial release was observed under speech masking compared to noise masking $(t(23)=13.58, p<.001$, Cohen's $d=3.90)$. More importantly, the spatial release was significantly larger for the unpleasant voice than the pleasant voice $(t(22)=5.91, p<.001$, Cohen's $d=1.78$ ). 
Speech Masking

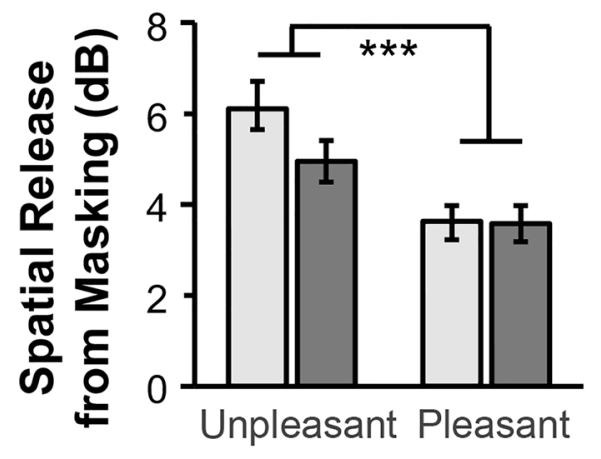

Noise Masking

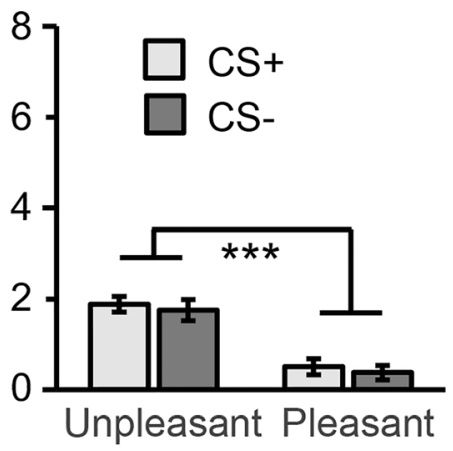

\section{Emotional Valance of Vocal Timbre}

Fig. 5 Comparison of the spatial release from masking between the pleasant and unpleasant voices under two masking types. Larger

These results suggest that the spatially-induced unmasking effect was increased for a target speaker with a negative vocal timbre compared to that with a positive vocal timbre, regardless of whether emotional conditioning is introduced or not and the masker type is different.

\section{Subjective emotional evaluation of vocal timbres}

For each experimental session with the speech-masking and noise-masking conditions, participants evaluated their subjective emotional feelings induced by the pleasant/unpleasant target-speaker voices at the end of the experiment. Thus, the emotional ratings for both voices were obtained twice, and the two ratings showed a significant correlation (for emotional valence, $r=0.791, p<.001$; for emotional arousal, $r=.807$, $p<.001$ ), reflecting the high test-retest reliability of participants' subjective emotional reports.

In the speech-masking session, the emotional valence of the pleasant voice $(5.48 \pm 1.39)$ was significantly higher than the unpleasant one $(4.26 \pm 0.93)(t(23)=3.42, p=.002$, Cohen's $d=.70)$. In the noise-masking session, the difference in emotional valence between the pleasant voice $(5.41 \pm 1.37)$ and the unpleasant voice $(4.50 \pm 1.45)$ reached the marginal significance $(p=.061)$. No significant difference was found between the emotional arousal between the two voices $(p>$ $.05)$. The emotional evaluation of the two target voices from 24 participants in this study was consistent with the results from 50 raters as described in the Methods, confirming the different emotional characteristics of the vocal timbre between the two target voices.

Note that participants gave their emotional ratings after they received emotional learning and speech-recognition tests. The effects of emotional learning on their subjective emotional ratings of the voices were analyzed. Independent-sample $t$ tests showed that the aversive conditioning significantly decreased the emotional valence of the unpleasant voice (in the speech-masking session, $t(22)=-2.34, p=.028$, Cohen's $d=$ spatial-induced unmasking effect was observed for the unpleasant vocal timbre compared to the pleasant one. Error bar represent SEs. ${ }^{* * *} p<.001$

.96; and in the noise-masking session, $t(22)=-2.42, p=.024$, Cohen's $d=.99$ ), but not that of the pleasant voice ( $p=.671$ and .542 , respectively).

Considering the similar finding of an enhanced spatial release of target speech from masking for the unpleasant voice, the relationship between the emotional valence of a target voice and the speech recognition of that voice was examined. Interestingly, a pronounced negative correlation between the spatial release of speech-recognition thresholds and the emotional valence of the unpleasant voice $(r=-.453, p=.026)$, but not that of the pleasant voice $(r=.129, p=.552)$, was found. That is, for the unpleasant voice, the more negative emotion that listeners perceived from the target vocal timbre, the larger spatial release of target speech from masking (Figure 6). These results further support the findings that negatively emotional characteristics embedded in the vocal timbre facilitate the processes of spatially induced speech unmasking.

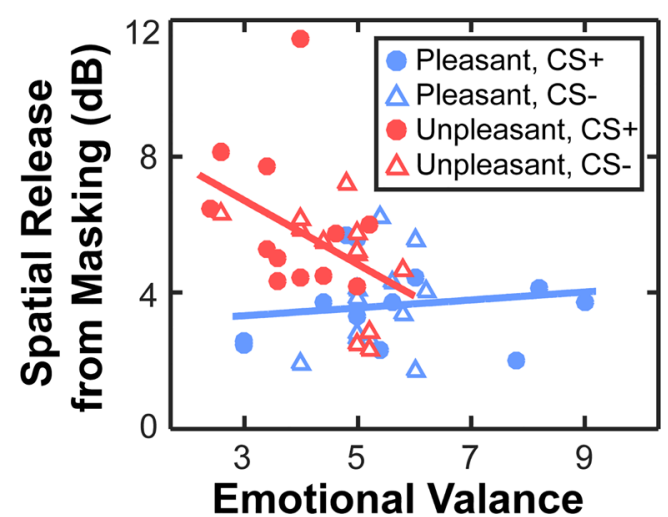

Fig. 6 Relationship between the subjective ratings of voice emotional valance and the spatial release from masking during target-speech recognition. For the unpleasant voice, the more negative participants rated on the emotional valance of the target voice, the larger the spatial-induced unmasking effect was during the speech-in-noise perception (red line, $r=$ $-.453, p=.026$ ). For the pleasant voice, no significant correlation was observed (blue line, $r=.129, p=.552$ ) 


\section{Discussion}

The present study examined the impact of emotional vocal timbre on speech unmasking under either a noise-masking or a speech-masking environment. The results showed that (1) target speech with a negative vocal timbre was better recognized compared to that with a positive timbre under a CS+ condition against the speech masker, and under both CS+ and CS- conditions against the noise masker; (2) aversively conditioning the negative voice significantly improved targetspeech recognition compared to the control learning under the speech-masking condition, but not under the noisemasking condition; (3) most importantly, the spatially induced speech-unmasking effect was larger for the target speaker with the negatively emotional vocal timbre compared to that with the positively emotional one (revealed by the difference of speech-recognition thresholds between different vocal timbres, and by the relationship between subjective emotional ratings of the vocal timbre and the amount of spatial-induced masking release). Based on these results, this study for the first time revealed that the emotional feature embedded in vocal timbres of the target speaker was crucial for the speechunmasking processing under the "cocktail-party" environment.

Previous studies have shown that the emotional prosody modulates speech recognition against to noisy backgrounds (Dupuis \& Pichora-Fuller, 2014; Gordon \& Hibberts, 2011; Gordon \& Ancheta, 2017). Specifically, negative emotion such as fearful prosody enhances the recognition of masked speech (Dupuis \& Pichora-Fuller, 2014), and the word recognition is improved with happily expressed prosody (Gordon \& Hibberts, 2011; Gordon \& Ancheta, 2017). This emotioninduced advantage of the speech-in-noise perception can be attributed to the low-level acoustics, which vary with affective prosody (Scherer, 1986) and facilitate the segregation between the target speech stream and the noisy scene (Bregman, 1994). Besides, the emotional features might enhance selective attention to the target auditory stream, thereby improving recognition of the target speech ( $\mathrm{Lu}$ et al., 2018). According to the taxonomy of affective sounds proposed by Frühholz, Trost, and Kotz (2016), affective sounds can be classified according to their potential perceivers. The source of affective meanings embedded in speech prosody originates from the sender, that is, emotional prosody conveys the emotional state of the speaker. However, the emotional feelings caused by the vocal timbre or by the aversive conditioning basically spring from the perceiver, depending on listener's personal experience or evolutionary responses. In this study, the target-speech voice was controlled and portrayed in the neutral prosody, but the listeners reported their own subjective emotional feelings based on speaker's vocal timbres. Consistent with previous findings of the emotional modulation on masked-speech recognition (Dupuis \& Pichora-Fuller, 2014; Lu et al., 2018), our study found that the speech with an emotionally negative vocal timbre was better tracked and recognized. Clearly, the current study provided the first evidence showing that affective signals embedded in the speaker's vocal timbre and perceived by the listener exert a strong unmasking influence over the speech perception against a masking background, particularly an informational masking background. The findings are complementary to previous findings that affective prosodies (Dupuis \& Pichora-Fuller, 2010, 2014; Gordon \& Hibberts, 2011; Gordon \& Ancheta, 2017; Iwashiro et al., 2013) or affective contents (Iwashiro et al., 2013) conveying emotional information have impacts on the noise-masked speech.

The present study used the two target voices with different fundamental frequencies in the experiment. The emotional valences and arousals induced by the vocal timbres were scored by 50 raters who did not participate in the formal experiment, and were also scored by 24 participants after they received emotional/neural learning for the vocal timbres. The consistent rating results on the emotional valences of the two target voices confirms the reliability of the differentiated emotional features embedded in their vocal timbres. Note that the different fundamental frequencies between the two target voices might also lead to different speech recognition against the same noisy background. However, the significant correlation between the spatial unmasking effect and the subjective ratings of the unpleasant vocal timbre, but not the pleasant vocal timbre, cannot be attributed to the fundamental frequencies, because the mean fundamental frequencies were kept the same for each target voice during the entire experiment. Therefore, negative emotional features embedded in the vocal timbre but not fundamental frequency contribute to the improvement of the speech-in-noise recognition. In future studies, more target voices with different fundamental frequencies should be included and their emotional features should also be analyzed to examine the additional effects of the fundamental frequency of voices on speech perception against noisy background.

In this study, two ways of emotional manipulations were introduced. Different emotional valences of the speakers' vocal timbres were naturally perceived by the listeners, and were further negatively modulated by the classical emotional learning. The results indicate that aversively conditioning the unpleasant voice can effectively enhance the target-speech recognition under the two-talker speech-masking condition, which is consistent with our previous studies ( $\mathrm{Lu}$ et al., 2018). Interestingly, compared to a two-talker speech background in which informational masking reaches the maximum level (Wu et al., 2007), the emotional-learning-induced unmasking effect disappeared under a steady-state speechspectrum noise-masking condition. It is known that emotional learning drives selective attention to objects with threatening signals (Koster, Crombez, Van Damme, Verschuere \& De Houwer, 2005; Pischek-Simpson, Boschen, Neumann \& 
Waters, 2009). After aversively conditioning, the negative emotion embedded in the vocal timbre was intensified and able to capture auditory attention to the target stream at a higher processing level, leading to better perceptual segregation of the target speech stream from the masker. In contrast, under the energetic-masking condition when the target speech was overwhelmed by the background noise at the peripheral level, such an attention-related advantage was reduced as listeners did not find it difficult to selectively attend to the target speech against the noise masker. Nevertheless, it should be noted that the enhanced unmasking effect caused by emotional conditioning was not significant for the pleasant voice in the present study, which might result from the emotional incongruence between the voice valence and aversive conditioning. It has been proposed that the neural activation of the auditory cortex was diminished when incongruent emotions (e.g., cross-modal happy-sad contrasts) were processed (Jeong et al., 2011). The emotional incongruence between the pleasant voice (emotionally positive) and the aversive conditioning (emotionally negative) may lead to impairment of auditory perception, hence distorting the speech-unmasking effect. In future, more work is needed to clarify the psychological and neural mechanisms underlying the interaction between emotional features of vocal timbres and experience-dependent learning in modulating the speech-in-noise perception.

In this study, the perceived spatial relationship between the target speech and the masker was manipulated in two conditions: separated and co-located. The results showed that the perceived separation between the target speech and the masker significantly increased the speech-in-noise recognition, which was consistent with previous findings (Arbogast et al., 2002; Freyman et al., 1999; Lu et al., 2018; Li et al., 2004; Schneider, Li \& Daneman, 2007; Wu et al., 2005). Furthermore, the spatially induced release of target speech from masking was modulated by the emotional characteristics of the target speaker's vocal timbre. More specifically, the unmasking effect caused by perceived spatial separation was larger for the unpleasant vocal timbre than that for the pleasant one. These results can be explained from the perspective of selective attention: Compared to the co-located condition, when the target and maskers were perceptually separated, the listeners were able to better allocate their attention to the target speech (location) and perceptually segregate the target speech from the competing background. For an unpleasant voice, the negative emotional feature of the vocal timbre further enhances this attention-guided unmasking processing, resulting in a larger spatial-induced unmasking for the target speech perception articulated by the emotionally negative vocal timbre. The findings of a larger spatial release of target speech from masking for the unpleasant voice than that for the pleasant voice suggested that different features in a speech stream (such as the spatial features and the emotional features) were processed simultaneously and interactively in the speech-in-noise perception. From the perspective of feature binding, which is a general brain function that applies to all types of knowledge representations (Von der Malsburg, 1999), an important cognitive process in solving the "cocktail-party" problem is to represent the integrations of features (Haykin \& Chen, 2005), and the dynamic binding is closely associated with the attention mechanism (Singer, 1993). Thus, the findings of this study are related to the mechanisms underlying both attention (possibly driven by emotional and spatial factors) and integration ("binding") of multiple features (cues) in a complex auditory scene. The dynamics underlying the interaction between multiple features in the speechunmasking processing and its relationship with auditory/ emotional attention should be further investigated in future studies.

\section{Conclusions}

An emotionally negative valence of the target vocal timbre is more associated with improvement of target-speech recognition compared to that of an emotionally positive vocal timbre under masking conditions. Emotionally conditioning the negative voice further enhances the target-speech recognition under the informational masking condition. The interaction between the perceived-spatial-separation unmasking cue and the emotional unmasking cue leads to larger enhancement of the spatially induced unmasking of the speech recognition for target speech with an emotionally negative vocal timbres than that with an emotionally positive vocal timbre.

Acknowledgements This work was supported by the National Natural Sciences Foundation of China (31771252).

\section{Compliance with ethical standards}

Conflicts of Interest None declared.

\section{References}

Arbogast, T. L., Mason, C. R., \& G. Kidd Jr (2002). The effect of spatial separation on informational and energetic masking of speech. Journal of the Acoustical Society of America, 112(1), 2086-2098.

Arons, B. (1992). A review of the cocktail party effect. Journal of the American Voice I/O Society, 12(7), 35-50.

Bradley, M. M., \& Lang, P. J. (1994). Measuring Emotion: The SelfAssessment Manikin and the Semantic Differential. J Behav Ther Exp Psychiatry, 25(1), 49-59.

Bradley, M. M., \& Lang, P. J. (2007). The International Affective Digitized Sounds (IADS-2): Affective ratings of sounds and instruction manual. University of Florida, Gainesville, FL, Tech. Rep. B-3, $29-41$

Bragman, A. S. (1994). Auditory scene analysis: The perceptual organization of sound. Cambridge: MIT Press. 
Brainard, H. D. (1997). The Psychophysics Toolbox. Spatial Vision, 10(4), 433-436.

Bregman, A. S. (1994). Auditory Scene Analysis: The Perceptual Organization of Sound. Cambridge: MIT Press.

Brungart, D. S. (2001). Informational and energetic masking effects in the perception of two simultaneous talkers. Journal of the Acoustical Society of America, 109(3), 1101-1109.

Case J., Seyfarth, S., and Levi, Susannah V. (2018). Short-term implicit voice-learning leads to a Familiar Talker Advantage: The role of encoding specificity. The Journal of the Acoustical Society of America, 144, EL479.

Cherry, E. C. (1953). Some experiments on the recognition of speech, with one and with 2 ears. Journal of the Acoustical Society of America, 25(5), 975-979. https://doi.org/10.1121/1.1907229

Dupuis, K., \& Pichora-Fuller, M. K. (2010). Use of Affective Prosody by Young and Older Adults. Psychology and Aging, 25(1), 16-29. https://doi.org/10.1037/a0018777

Dupuis, K., \& Pichora-Fuller, M. K. (2014). Intelligibility of Emotional Speech in Younger and Older Adults. Ear and Hearing, 35(6), 695707.

Eastwood, J. D., Smilek, D., \& Merikle, P. M. (2001). Differential attentional guidance by unattended faces expressing positive and negative emotion. Perception \& Psychophysics, 63(6), 1004-1013.

Koster, E., Crombez, G., Van Damme, S., Verschuere, B., De Houwer, J. (2005). Signals for threat modulate attentional capture and holding: Fear-conditioning and extinction during the exogenous cueing task. Cognition \& Emotion, 19(5):771-780.

Fox, E. (2002). Processing emotional facial expressions: The role of anxiety and awareness. Cognitive, Affective, \& Behavioral Neuroscience 2(1), 52-63.

Freyman, R. L., Balakrishnan, U., \& Helfer, K. S. (2001). Spatial release from informational masking in speech recognition. Journal of the Acoustical Society of America, 109(5), 2112-2122.

Freyman, R. L., Helfer, K. S., McCall, D. D., \& Clifton, R. K. (1999). The role of perceived spatial separation in the unmasking of speech. Journal of the Acoustical Society of America, 106(6), 3578-3588.

Frühholz, S., Trost, W., \& Kotz, S. A. (2016). The sound of emotions: Towards a unifying neural network perspective of affective sound processing. Neuroscience \& Biobehavioral Reviews, 68, 96-110.

Gordon, M. S., \& Ancheta, J. (2017). Visual and acoustic information supporting a happily expressed speech-in-noise advantage. Quarterly Journal of Experimental Psychology, 70(1), 163-178.

Gordon, M. S., \& Hibberts, M. (2011). Audiovisual speech from emotionally expressive and lateralized faces. Quarterly Journal of Experimental Psychology, 64(4), 730-750.

Grandjean, D., Sander, D., Pourtois, G., Schwartz, S., Seghier, M. L., Scherer, K. R., \& Vuilleumier, P. (2005). The voices of wrath: brain responses to angry prosody in meaningless speech. Nature Neuroscience, 8(2), 145-146.

Haykin, S., \& Chen, Z. (2005). The Cocktail Party Problem. Neural Computation, 17(9), 1875-1902. https://doi.org/10.1162/ 0899766054322964

Helfer, K. S. (1997). Auditory and auditory-visual perception of clear and conversational speech. Journal of Speech, Language, and Hearing Research, 40, 432-443.

Holmes, E., Domingo, Y., \& Johnsrude, I. S. (2018). Familiar Voices Are More Intelligible, Even if They Are Not Recognized as Familiar. Psychological Science, 29(10), 1575-1583. https://doi.org/10.1177/ 0956797618779083.

Huang, Y., Huang, Q., Chen, X., Wu, X.-H., Li, L. (2009). Transient auditory storage of acoustic details is associated with release of speech from informational masking in reverberant conditions. Journal of Experimental Psychology: Human Perception and Performance, 35, 1618-1628.
Huang, Y., Xu, L.-J., Wu, X.-H., Li, L. (2010). The effect of voice cuing on releasing speech from informational masking disappears in older adults. Ear and Hearing, 31, 579-583.

Iwashiro, N., Yahata, N., Kawamuro, Y., Kasai, K., \& Yamasue, H. (2013). Aberrant Interference of Auditory Negative Words on Attention in Patients with Schizophrenia. PLOS ONE, 8(12), 9. https://doi.org/10.1371/journal.pone.0083201

Jeong, J. W., Diwadkar, V. A., Chugani, C. D., Sinsoongsud, P., Muzik, O., Behen, M. E., ... Chugani, D. C. (2011). Congruence of happy and sad emotion in music and faces modifies cortical audiovisual activation. Neuroimage, 54(4), 2973-2982. https://doi.org/10.1016/ j.neuroimage.2010.11.017

Johnsrude, I. S., Mackey, A., Hakyemez, H., Alexander, E., Trang, H. P. , \& Carlyon, R. P. . (2013). Swinging at a cocktail party: voice familiarity aids speech perception in the presence of a competing voice. Psychological Science, 24(10), 1995-2004.

Klaus R. Scherer, K. R. (1986). Vocal affect expression: A review and a model for future research. Psychological Bulletin, 99(2):143-165.

Pischek-Simpson, L. K., Boschen, M. J., Neumann, D. L., Waters, A. M. (2009). The development of an attentional bias for angry faces following Pavlovian fear conditioning. Behaviour Research and Therapy 47(4):322-330

Levi, S. V., Winters, S. J., \& Pisoni, D. B. (2011). Effects of crosslanguage voice training on speech perception: Whose familiar voices are more intelligible? Journal of the Acoustical Society of America, 130(6), 4053-4062.

Li, H.-H., Kong, L.-Z., Wu, X.-H., Li, L. (2013). Primitive auditory memory is correlated with spatial unmasking that is based on direct-reflection integration. PLOS ONE, 8 (4) e63106.

Li, L., Daneman, M., Qi, J. G., \& Schneider, B. A. (2004). Does the Information Content of an Irrelevant Source Differentially Affect Spoken Word Recognition in Younger and Older Adults? Journal of Experimental Psychology Human Perception \& Performan, 30(6), 1077-1091.

Lu, L., Bao, X., Chen, J., Qu, T., Wu, X., \& Li, L. (2018). Emotionally conditioning the target-speech voice enhances recognition of the target speech under "cocktail-party" listening conditions. Attention Perception \& Psychophysics, 80(4), 871-883.

New, J. J., \& German, T. C. (2015). Spiders at the cocktail party: an ancestral threat that surmounts inattentional blindness. Evolution \& Human Behavior, 36(3), 165-173.

Nygaard, L. C., \& Pisoni, D. B. (1998). Talker-specific learning in speech perception. Perception \& Psychophysics, 60(3), 355-376.

Ohman, A., Flykt, A., \& Esteves, F. (2001). Emotion Drives Attention : Detecting the Snake in the Grass. Journal of Experimental Psychology General, 130(3), 466-478.

Pollack, I., Pickett, J. M., \& Sumby, W. H. (1954). ON THE IDENTIFICATION OF SPEAKERS BY VOICE. Journal of the Acoustical Society of America, 26(3), 403-406. https://doi.org/10. 1121/1.1907349

Freyman, R. L., Balakrishnan, U., \& Helfer, K. S. (2004). Effect of number of masking talkers and auditory priming on informational masking in speech recognition. The Journal of the Acoustical Society of America, 115(5):2246-2256.

Sander, D., Grandjean, D., Pourtois, G., Schwartz, S., Seghier, M. L., Scherer, K. R., \& Vuilleumier, P. (2005). Emotion and attention interactions in social cognition: Brain regions involved in processing anger prosody. Neuroimage, 28(4), 848-858.

Schneider, B. A., Li, L., \& Daneman, M. (2007). How competing speech interferes with speech comprehension in everyday listening situations. Journal of the American Academy of Audiology, 18(7), 559572. https://doi.org/10.3766/jaaa.18.7.4

Singer, W. (1993). Synchronization of cortical activity and its putative role in information-processing and learning. Annual Review of Physiology, 55, 349-374. 
Spreadborough, K. L., \& Anton-Mendez, I. (2018). It's not what you sing, it's how you sing it: How the emotional valence of vocal timbre influences listeners' emotional perception of words. Psychology of Music.

von der Malsburg, C. (1999). The what and why of binding: The modeler's perspective. Neuron, 24(1), 95-104. https://doi.org/10.1016/ s0896-6273(00)80825-9

Vuilleumier, P. (2005). How brains beware: neural mechanisms of emotional attention. Trends in Cognitive Sciences 9(12), 585-594.

Wallach, H., Newman, E. B., \& Rosenzweig, M. R. (1949). A Precedence Effect in Sound Localization. The Journal of the Acoustical Society of America, 21, 468.

Wolfram, S. (1991). Mathematica: A system for doing mathematics by computer. Addison-Wesley, New York.
Wu, X., Wang, C., Chen, J., Qu, H., \& Li, W. (2005). The effect of perceived spatial separation on informational masking of Chinese speech. Hear Res, 199(1-2), 1-10.

Wu, X., Chen, J., Yang, Z., Huang, Q., Wang, M., \& Li, L. (2007). Effect of number of masking talkers on speech-on-speech masking in Chinese. In Proceedings of Interspeech (pp. 390-393). Antwerp, Belgium.

Yang, Z. G., Chen, J., Huang, Q., Wu, X. H., Wu, Y. H., Schneider, B. A., \& Li, L. (2007). The effect of voice cuing on releasing Chinese speech from informational masking. Speech Communication, 49(12), 892-904. https://doi.org/10.1016/j.specom.2007.05.005

Publisher's note Springer Nature remains neutral with regard to jurisdictional claims in published maps and institutional affiliations. 